\title{
On the Simulation of Offshore Oil Facilities at the System Level
}

\author{
Joris Costes $^{1,2} \quad$ Jean-Michel Ghidaglia $^{2} \quad$ Philippe Muguerra $^{3} \quad$ Keld Lund Nielsen $^{4}$ \\ Xavier Riou $^{3}$ Jean-Philippe Saut ${ }^{1} \quad$ Nicolas Vayatis $^{2}$ \\ ${ }^{1}$ Eurobios SCB, 86 avenue Lénine, 94250 Gentilly, France \\ \{joris.costes, jpsaut\}@eurobios.com \\ ${ }^{2}$ CMLA, ENS Cachan, UMR 8536 CNRS, 61 avenue du président Wilson, 94235 Cachan cedex, France \\ \{jmg, vayatis\}@cmla.ens-cachan.fr \\ 3 eni saipem, 7 avenue de San Fernando, 78180 Montigny-le-Bretonneux, France \\ \{Philippe.MUGUERRA,Xavier.RIOU\} @ saipem.com \\ ${ }^{4}$ eni spa, exploration \& production division, San Donato Milanese (Mi), Italy \\ Keld.Lund.Nielsen@eni.com
}

\begin{abstract}
Offshore oil facilities are complex systems that involve elaborate physics combined with stochastic aspects related, for instance, to failure risk or price variation. Although there exist many dedicated software tools to simulate flows typically encountered in oil exploitations, there is still no tool that combines physical (mostly engineering fluid mechanics) and risk simulation. Such a tool could be useful to engineers or decision makers for specification, design and study of offshore oil facilities. We present a first step towards the creation of such a tool. Our current simulator is based on new Modelica components to simulate fluid flows and on stochastic simulation at a higher level, for modeling risk and costs. Modelica components implement physical models for single and two-phase flows in some typical devices of an offshore field. The risk simulation uses Markov chains and statistical indicators to assess performance and resilience of the system over several months or years of operation.
\end{abstract}

Keywords: fluid flow; two-phase flow; risk estimation; Monte Carlo simulation

\section{Introduction}

With the increasing rarity of readily accessible reservoirs, as oil has to be extracted from deeper undersea, capital investments and risks associated to offshore oil facilities become higher and higher. In this context, a careful evaluation of cost and risk represents a crucial step in the conception of a new offshore facility. This evaluation must be performed at the system level because it involves phenomena of very different na- tures and scales, that can interact together. We identify three main types of phenomena to be taken into account in the simulation: Physical, failure-related (risk) and economic (cost). The physical phenomena obviously include fluid flow through common components (pipes, tanks, valves, etc.) but can also include various other aspects like e.g. strength of materials, heat transfer or chemistry of wax or hydrate formation. The failure phenomena are the discrete-time events due to specific (extreme) conditions (e.g. break due to component fatigue) or to accidental situations. Simulating failures is useful to evaluate the potential risks of a particular architecture/design. The economic phenomena encompass all that is related to cost or price but does not directly depends on physics (unlike e.g. the oil production). For instance the oil barrel price, the price of pipe wall material (typically steel) or the transportation cost. All these variables directly impact the profitability of an exploitation.

This paper presents our first effort in the conception of a system-level offshore facility simulation. Before starting the work, it was necessary to point out simulation requirements and expected difficulties. Requirements are more related to the user point-of-view while difficulties are more related to the physics of an offshore oil facility:

- Reasonable computation times are preferable since we want the simulator to be usable as a design tool,

- Complexity: An offshore exploitation can involve dozens or hundreds of elementary components,

- Modularity: A model is meant to be built by as- 
sembling elementary components of typical parts of an offshore field,

- Accurate prediction of highly-turbulent fluid flow is not possible (typical Reynolds number in oil \& gas industry flows is $>10000$ ),

- Many physical parameters are known only in certain ranges or even uncertain,

- Experimental validation is difficult or limited to existing literature.

Henceforth it was decided to build first physical models with moderate complexity in order to follow the large-scale behavior of the real components within reasonable computation time. Another choice was to decompose the simulation into two different layers: One for the physics and one for the cost/risk estimation. The physics layer models deterministic phenomena only, while the cost/risk layer is based on probabilities to model risk and uncertainty. The physical simulation takes advantage of the flexibility and modularity that are possible with Modelica features (object-oriented, acausality). Other authors have used a similar approach of employing a stochastic simulation layer on top of a Modelica-based simulation. For instance in the context of Building Performance Simulation [14] to model weather and room occupancy as stochastic processes. Propositions were recently submitted by Bouskela et al. [3] to enrich the Modelica language with the possibility to define uncertain variables with user-configurable probability law. The authors exposed some applications in power plant or combustion engine field to perform data reconciliation or uncertainty propagation. With the current Modelica version, the authors had to rely on the external program OpenTURNS [7] to compute uncertainty propagation on a fluid pipe system example. It seems presently unavoidable to work with this kind of architecture (Modelica + external program with an interface layer) since stochastic modeling is clearly out of the scope and objectives of the current Modelica specifications.

The paper is divided as follows. Section 2 focuses on fluid flow simulation. It describes the hypotheses and equations used to build the Modelica components of the offshore facility. Section 3 deals with the estimation of cost and risk. It details the different variables of interest and their stochastic model (Markov chain representation and Monte Carlo simulation). Section 4 presents some first results obtained on a simplified architecture used as a proof of concept.

\section{Simulation of flows in an offshore oil facility}

The first choice when conceiving the fluid flow simulation was to decide the accuracy level that would give the appropriate balance between fast computation time and physical coherence. To ensure the computational tractability of the models we have chosen 1D or OD models depending on the component. This for permanent-regime study. The latter choice comes from the considered time scale that is rather large in order to estimate typical daily production (hours extrapolated to a full day). In the current development stage, we are not yet concerned with heat transfer so isothermal transformations are assumed. The fluid that flows from an oil reservoir is usually a mixture of oil and gas (in particular because gas might be injected inside the reservoir to increase the flow rate of production). The basic connector used in our simulation is then defined by three parameters $(p, q, \varphi)$ :

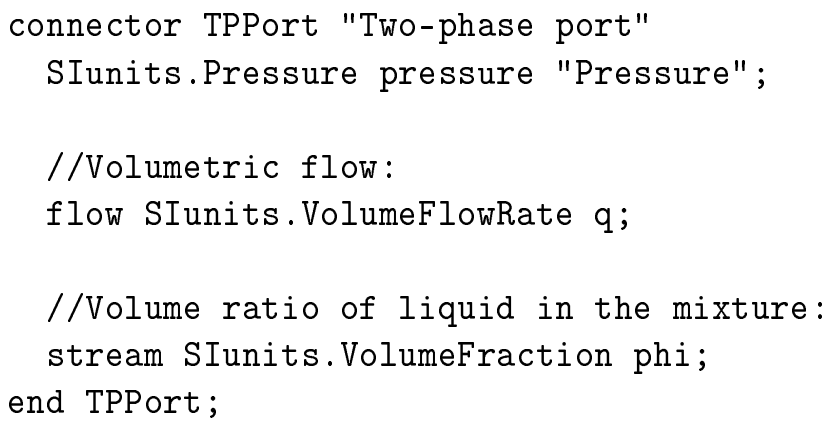

In addition to the permanent-regime assumption, we assume that fluids are incompressible with exceptions for a few components (e.g. the oil-gas separator in Section 2.4). The aforementioned assumptions are more restrictive than the ones of the Fluid library [4] in the Modelica Standard Library that is more generic, at the price of a higher computation time.

\subsection{Single-phase flow in a pipe}

The model of single-phase flow in a pipe is encountered only in limited areas of the offshore field, e.g. after an oil-gas separator. It is however useful in order to compute the virtual single-phase pressure drop used in the two-phase pipe model (Section 2.2). It requires a variant of the TPPort connector where $\varphi$ (phi) is removed. The fluid velocity $v$ is computed from the volume flow rate $q$ and the pipe cross-sectional area $A$ :

$$
\mathrm{v}=\frac{q}{A}
$$


Since we have volume flow conservation, $q=q_{a}=q_{b}$ where $q_{a}$ and $q_{b}$ are the values of $q$ in the two connectors at the ends of the pipe.

Reynolds number is

$$
R e=\frac{\rho D_{h}|\mathrm{v}|}{\mu},
$$

where $\rho$ is the fluid density, $D_{h}$ the pipe hydraulic diameter and $\mu$ denotes dynamic viscosity of the fluid.

The frictional pressure loss is computed as

$$
\Delta p= \pm f_{d} \frac{L}{D_{h}} \rho \frac{\mathrm{v}^{2}}{2}
$$

where $f_{d}$ is the Darcy friction factor and $L$ the pipe length. The sign of $\Delta p$ must be chosen so that the pressure decreases in the direction of the flow.

The Darcy friction factor $f_{d}$ depends on the flow regime. As only turbulent flows are encountered in the considered oil and gas applications, only the turbulent regime is of interest. For this regime, among the many existing correlations, we chose Haaland's formula [9]:

$f_{d_{\text {turbulent }}}=\left(-1.8 \log _{10}\left(\frac{6.9}{\operatorname{Re}}+\left(\frac{k_{s}}{3.7 D_{h}}\right)^{1.11}\right)\right)^{-2}$,

where $k_{s}$ is the roughness height that characterizes the rugosity of the pipe inner wall. It is typically between $1 \mu \mathrm{m}$ and $1 \mathrm{~mm}$.

\subsection{Two-phase flow in a pipe}

Several Modelica models have been proposed to deal with two-phase flow modeling $[1,6,10,2]$, with applications to steam generators or refrigerators. These models are centered on accurate simulation (1D, boundary model) of a few components. To simulate an offshore field architecture, since we are for now only concerned with the related evolution of pressure loss and flow rate, we chose a much simpler model with very low computational requirements, based on the work by Lockhart and Martinelli [12]. Lockhart and Martinelli proposed a correlation to compute the pressure drop of a two-phase mixture in a pipe, from the pressure drops computed for the two (virtual) singlephase flows. Chisholm [5] gave some theoretical basis for the correlation and recommended a simplified version of the formula, for engineering calculations:

$$
\Delta p_{T P}= \pm\left(\Delta p_{L}+C \sqrt{\left\|\Delta p_{L} \Delta p_{G}\right\|}+\Delta p_{G}\right),
$$

where:
- $\Delta p_{T P}$ is the pressure drop for the two-phase mixture,

- $d \Delta p_{L}$ is the pressure drop as if the liquid flowed alone,

- $d \Delta p_{G}$ is the pressure drop as if the gas flowed alone,

- $C$ is a correction coefficient which depends on the flow type of each phase (see Table 1). In practice, only the turbulent-turbulent case is of interest.

The sign of $\Delta p$ must be chosen so that the pressure decreases in the direction of the flow.

\begin{tabular}{|c|c|c|}
\hline \multicolumn{2}{|c|}{ Flow regime } & \\
\hline Liquid & Gas & Coefficient $C$ \\
\hline turbulent & turbulent & 20 \\
\hline laminar & turbulent & 12 \\
\hline turbulent & laminar & 10 \\
\hline laminar & laminar & 5 \\
\hline
\end{tabular}

Table 1: Coefficient $C$ for two-phase pressure drop computation (from [5]).

Note that the library FluidDissipation[16] also refers to the work of Chisholm, in a more complete implementation ${ }^{1}$.

\subsection{Junctions}

They are components used to direct the flows in two pipes into a single one. The junctions can be for instance used to inject gas in a liquid flow in order to increase its flow rate. When the two mixing fluids are in the same phase, a simple model can be used that just averages their characteristics. When the two phases are different a finer model is necessary. We consider here the cases of horizontal and vertical junctions.

\subsubsection{Horizontal junction}

The configuration of Figure 1 is considered. $\left(p_{1}, \mathrm{v}_{1}, \rho_{1}\right), \quad\left(p_{2}, \mathrm{v}_{2}, \rho_{2}\right)$ and $\left(p_{3}, \mathrm{v}_{3}, \rho_{3}\right)$ are the pressure, velocity and density at the liquid inlet, gas inlet and mixture outlet respectively,

Fluid velocities are $\mathrm{v}_{1}=\frac{q_{1}}{A}, \mathrm{v}_{2}=\frac{q_{2}}{A_{g}}$ and $\mathrm{v}_{3}=\frac{q_{3}}{A}$ where $A$ is the cross-section area of the liquid inlet and mixture outlet and $A_{g}$ is the cross-section area of the gas

\footnotetext{
${ }^{1}$ http://xrg-simulation.de/en/products/xrg-library/xrgfluiddissipation-library
} 


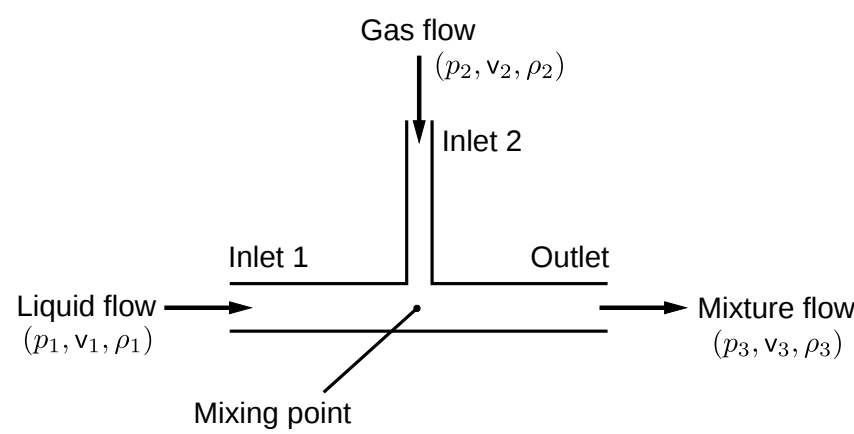

Figure 1: Schematic of the horizontal two-phase junction.

inlet. Conservation of both mass and volume flows gives:

$$
\begin{aligned}
\varphi & =\frac{\mathrm{v}_{1} A}{\mathrm{v}_{1} A+\mathrm{v}_{2} A_{g}}, \\
\rho_{3} & =\varphi \rho_{1}+(1-\varphi) \rho_{2}, \\
\mathrm{v}_{3} & =-\left(\mathrm{v}_{1} A+\mathrm{v}_{2} A_{g}\right) / A .
\end{aligned}
$$

From Ji et al. [11] we took the empirical equation for the momentum correction coefficient $K$ that is computed from the momentum flux ratio $M$. The equations are:

$$
\begin{array}{r}
M=\frac{\rho_{1} \mathrm{v}_{1}^{2}}{\rho_{2} \mathrm{v}_{2}^{2}}, \\
K=1+0.256 M^{0.223}, \\
p_{1}-p_{3}=K\left(\rho_{3} \mathrm{v}_{3}^{2}-\rho_{1} \mathrm{v}_{1}^{2}\right)
\end{array}
$$

Note that we use here the most generic formula in [11], because the roles of gas (side inlet) and liquid (front inlet) are swapped compared to what is in the paper. At this point, the system is under-determined since $p_{2}$ does not appear in any of the above equations (it would if the junction angle was $\neq 90^{\circ}$ ) so we need an extra equation. We choose the assumption that both inlet 1 and inlet 2 are close enough to the mixing point in order to have

$$
p_{1}=p_{2}
$$

\subsubsection{Vertical junction}

A vertical junction is a horizontal junction rotated with a $90^{\circ}$ angle (Figure 2). Compared to the horizontal junction, a correction term is added to take into account the weight of the fluid:

$$
p_{1}-p_{3}=K\left(\rho_{3} \mathrm{v}_{3}^{2}-\rho_{1} \mathrm{v}_{1}^{2}+0.5 \operatorname{Lg}\left(\rho_{1}+\rho_{3}\right)\right) \text {, }
$$

where $g$ is the gravitational acceleration and $L$ the junction length.

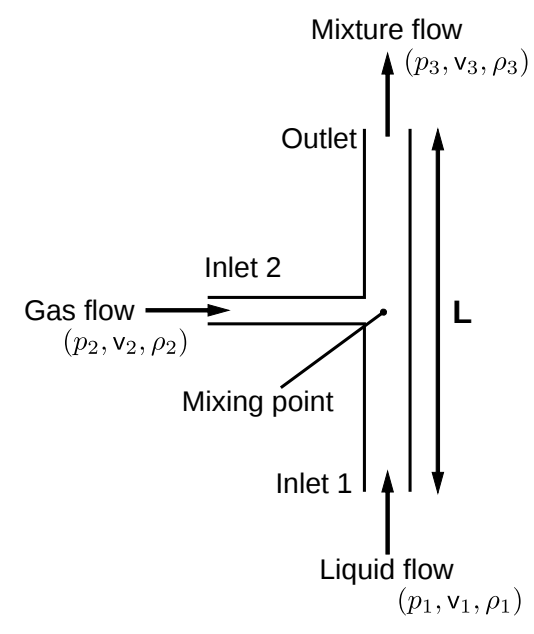

Figure 2: Schematic of the vertical two-phase junction.

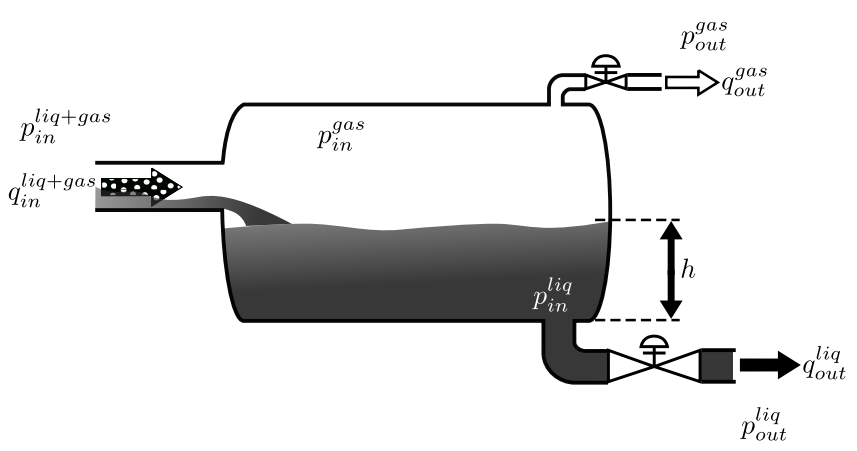

Figure 3: Schematic of the oil-gas separator.

\subsection{Separator}

The purpose of an oil-gas separator is to output two single-phase flows (one of liquid and one of gas) from one two-phase inflow (liquid+gas) (Figure 3). The studied separator dissociates the two phases by gravity, inside a tank or vessel. The physical input to the separator is the volume flow of oil-gas mixture that goes into the tank, while the outputs are the volume flows out of the tank. The device contains control loops to maintain the liquid level and the inside gas pressure at desired reference values. The oil-gas separator is consequently modeled as a controlled system. The two controlled values are the height of oil in the tank (denoted by $h$ ) and the gas pressure inside the tank (denoted by $p_{i n}^{\text {gas }}$ ). Internal sensors (supposedly perfect) measure both $h$ and $p_{i n}^{\text {gas }}$ so that they can be compared to their respective reference values $h_{\text {set }}$ and $p_{\text {set }}$. There is one control loop for each of $h$ and $p_{i n}^{g a s}$ and some variables are involved in the two control loops [8] (Figure 4).

The controllers are simple PIDs (ProportionalIntegral-Derivative). Transmission lines conduct the 


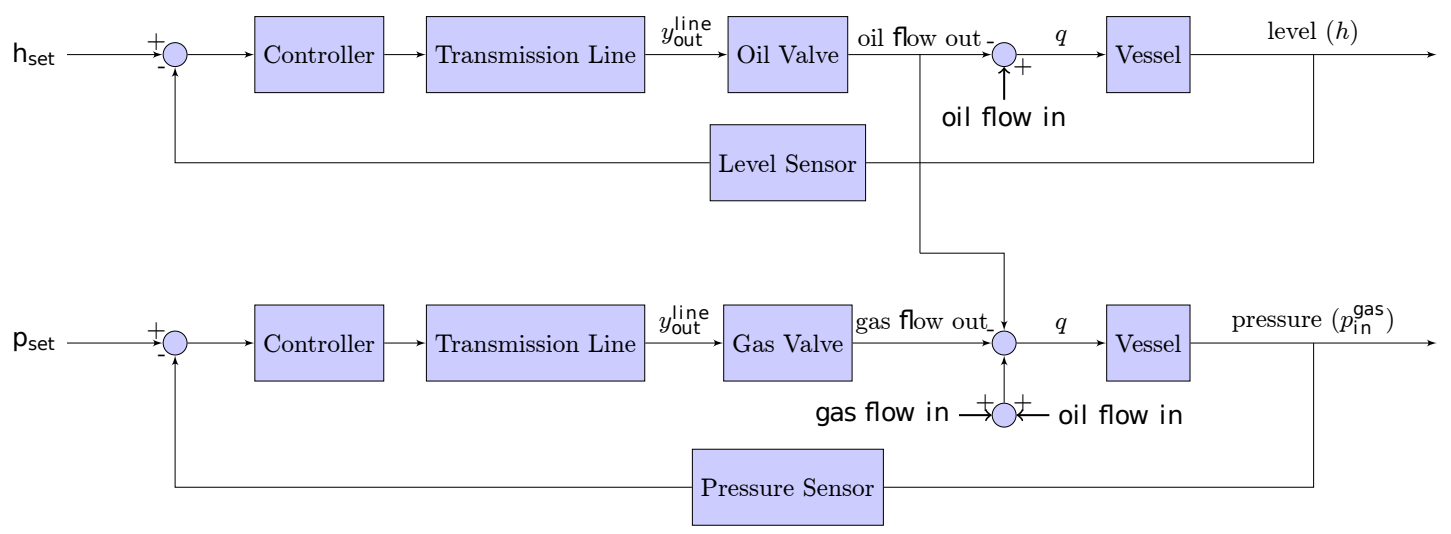

Figure 4: Liquid level and gas pressure control loops.

controller outputs to the control valves. They are modeled as first-order low-pass filters [8]. The valve aperture control parameter $\sigma_{\text {valve }}$ is the line signal $y_{\text {out }}^{\text {line }}$ after a $[0 ; 1]$ saturation is applied:

$$
\sigma_{\text {valve }} \in[0 ; 1]=\max \left(0, \min \left(y_{\text {out }}^{\text {line }}, 1\right)\right) .
$$

We give more details on each control loop in next sections.

\subsubsection{Level loop}

The vessel is a horizontal cylinder with diameter $d_{\varnothing}$, radius $r$ and length $L$. Let us introduce the distance $\tilde{h}$ from the liquid height $h$ to the vessel middle (i.e., $\mathrm{h}=r$ ):

$$
\tilde{h}= \begin{cases}h & \text { if } h \leq r, \\ d_{\varnothing}-h & \text { if } h>r .\end{cases}
$$

The liquid level $h$ is then related to liquid volume flows according to

$$
q_{\text {in }}^{l i q}-q_{\text {out }}^{l i q}=2 \sqrt{d_{\varnothing} \tilde{h}-\tilde{h}^{2}} L \frac{d h}{d t},
$$

where

$$
q_{i n}^{l i q}=\varphi q_{i n}^{l i q+g a s} .
$$

Finally $q_{\text {out }}^{\text {liq }}$ is related to $p_{\text {in }}^{\text {liq }}-p_{\text {out }}^{\text {liq }}$ with a friction law like in Section 2.1, which is denoted $\mathrm{f}_{\text {fric }}()$. The factor $\sigma_{\text {valve }}$ is added because we assume the valve aperture to act linearly on the flow:

$$
q_{\text {out }}^{\text {liq }}=\sigma_{\text {valve }} \mathrm{f}_{\text {fric }}\left(p_{\text {in }}^{\text {liq }}-p_{\text {out }}^{\text {liq }}\right) .
$$

\subsubsection{Pressure loop}

The gas volume flows are:

$$
q_{\text {in }}^{\text {gas }}=(1-\varphi) q_{\text {in }}^{\text {liq }+ \text { gas }},
$$

$$
q_{\text {out }}^{\text {gas }}=\sigma_{\text {valve }}^{\prime} \mathrm{f}_{\text {fric }}^{\prime}\left(p_{\text {in }}^{\text {gas }}-p_{\text {out }}^{\text {gas }}\right),
$$

where $\sigma_{\text {valve }}^{\prime}$ is the gas valve aperture and $\mathrm{f}_{\text {fric }}^{\prime}()$ a friction function like in Section 2.1. The ideal gas law in the vessel gives:

$$
p_{\text {in }}^{\text {gas }} V^{\text {gas }}=n^{\text {gas }} R T,
$$

where $V^{\text {gas }}$ is the volume of gas inside the tank (above the liquid), $n^{\text {gas }}$ is the gas mass quantity (moles) inside the vessel, $R$ the ideal gas constant, and $T$ the temperature inside the tank.

Differentiating Eq.(20), while replacing $V^{\text {gas }}$ with $\left(V^{\text {total }}-V^{\text {liq }}\right)$, leads to:

$$
\begin{aligned}
& \underbrace{\left(V^{\text {total }}-V^{\text {liq }}\right)}_{V^{\text {gas }}} \frac{d p_{\text {in }}^{\text {gas }}}{d t}-p_{\text {in }}^{\text {gas }} \frac{d V^{\text {liq }}}{d t}= \\
& R T\left(q_{\text {in,mass }}^{\text {gas }}-q_{\text {out }, \text { mass }}^{\text {gas }}\right),
\end{aligned}
$$

where $V^{\text {total }}$ is the tank volume, $V^{\text {liq }}$ the volume occupied by the liquid, $q_{\text {in,mass }}^{\text {gas }}$ and $q_{\text {out }, \text { mass }}^{\text {gas }}$ are the gas mass inflow and outflow respectively. The tank volume is

$$
V^{\text {total }}=\pi r^{2} L \text {. }
$$

Volume $V^{l i q}$ is computed from the liquid height by introducing

$$
V_{t m p}=L\left(r^{2} \arccos \left(1-\frac{\tilde{h}}{r}\right)-(r-\tilde{h}) \sqrt{2 r \tilde{h}-\tilde{h}^{2}}\right),
$$

$$
V^{l i q}= \begin{cases}V_{t m p} & \text { if } h \leq r \\ V_{\text {total }}-V_{t m p} & \text { otherwise }\end{cases}
$$

The mass flows are computed from the ideal gas law (also assuming $p_{\text {in }}^{\text {liq }+ \text { gas }}=p_{\text {in }}^{\text {gas }}$ ):

$$
\begin{aligned}
q_{\text {in,mass }}^{\text {gas }} & =q_{\text {in }}^{\text {gas }} \frac{p_{\text {in }}^{\text {liq }+ \text { gas }}}{R T}, \\
q_{\text {out }, \text { mass }}^{\text {gas }} & =q_{\text {out }}^{\text {gas }} \frac{p_{\text {in }}^{\text {gas }}}{R T}
\end{aligned}
$$


Figure 5 shows the system response when the separator receives a sequence of inflow steps.
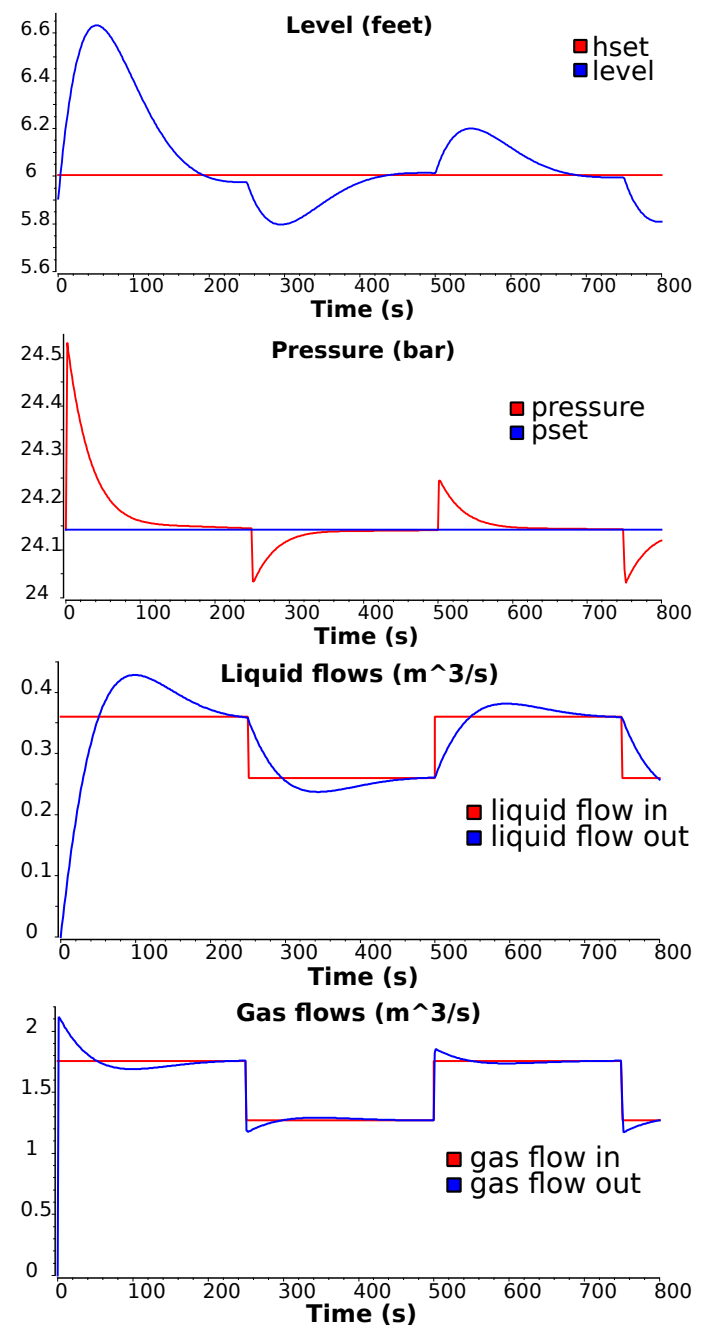

Figure 5: Responses to a square-wave input flow. The system shows a second-order behavior (overshooting and pseudo-oscillations).

\subsection{Other models}

Other models are necessary to simulate an offshore field. For instance, valves, pumps or tanks. The models are not described in detail here due to lack of space. They are chosen as simple as possible at this stage of the work. Hence ideal behavior is assumed:

- Valve: Two ports: port_a, port_b, one parameter: open=aperture signal:

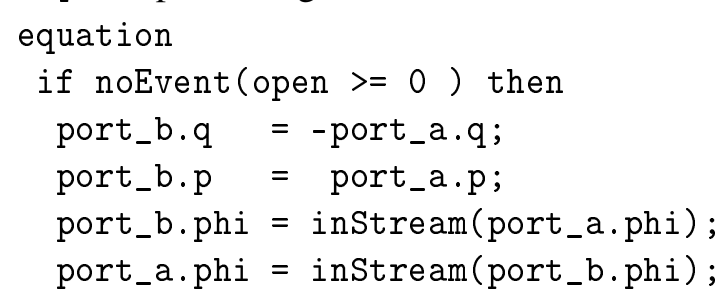

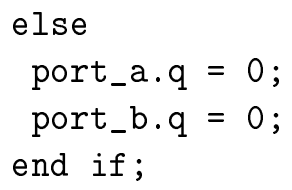

- Pump: Two ports: port_a, port_b, one parameter: $\mathrm{q} 0=$ imposed flow rate:

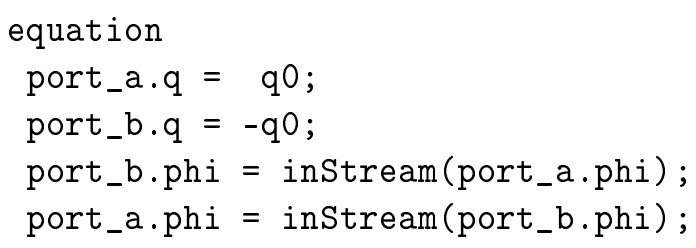

- Tank: One port: port_in, one parameter: $\mathrm{A}=$ horizontal area, one variable: $\mathrm{h}=$ liquid level: equation //hydrostatic pressure: port_in.p $=$ rho $* \mathrm{~g} * \mathrm{~h}$; $\operatorname{der}(h)=$ port_in.q/A;

\section{Estimation of costs and risks}

Estimating costs and risks requires a different modeling level than fluid flow simulation since it depends on exogenous factors, possibly stochastic (oil market, steel market, weather), or endogenous stochastic factors (failures). Dividing the simulation in two layers does not mean however that the layers are uncoupled. There is instead a strong dependency between them. For instance, the production income depends both on the extracted oil volume, computed in the physical simulation with an additional random perturbation term, and on the oil market and system state, the latter being modified by possible failures. Next sections describe the stochastic modeling of the facility. As further explained in Section 4, this part is not performed with Modelica but interacts with the Modelica simulation used for fluid flow modeling.

\subsection{Stochastic model of the offshore oil facil- ity}

\subsubsection{Markov chain model}

The system is assumed to be a Markovian process (i.e., memoryless) with discrete time. The time step is long (e.g. day or week) compared to the physical simulation time. The Markovian process is modeled as a Markov chain i.e., a finite state machine with transitions described as conditional probabilities. Each state of the chain corresponds to a particular operation state 


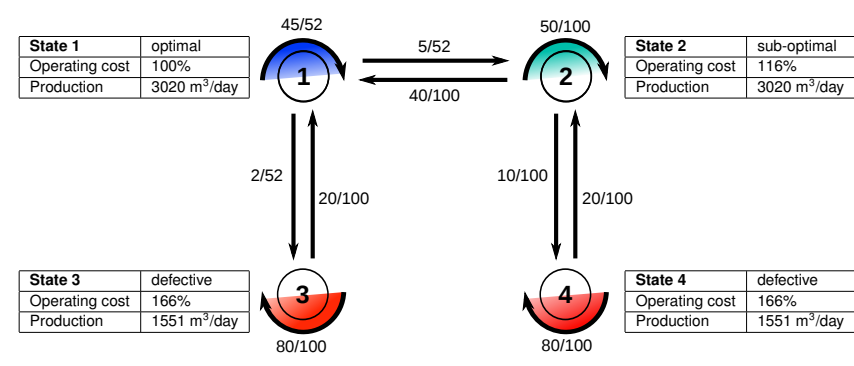

Figure 6: Markov chain representation of the offshore field with four possible operation states. The operating costs are given as percentages of the cost when in optimal state (state 1).

of the oil facility. Production volume of each state is computed with the physical simulation. It means that as many physical simulations will be conducted as the number of defined system states. The Markov chain requires the definitions of the possible transitions between states and their respective probability. These definitions are typically obtained from experimental data or expert knowledge. Figure 6 shows an example of Markov chain for four defined states with their associate operating cost and production volume. The Markov chain is used to simulate virtual life-cycles of the field under the randomness hypotheses on the events (price change, failure) that may occur over time. One such virtual cycle corresponds to one realization of randomness. Repeating many simulations allows to build empirical distributions of the output of interest (production, cost, resilience performance, etc.). This is the principle of Monte Carlo simulation.

\subsubsection{Monte Carlo simulation}

The goal of Monte Carlo simulation is to derive statistics from repeated simulations. With the Markov chain model, it means computing the evolution of the system state by random selection of the transition at each time step. The transition selection depends on the previously-defined transition probabilities. We now present some of the statistical quantities that are interesting in the context of offshore field simulation.

\subsection{Assessment indicators}

Various indicators can be calculated to obtain information about the system performance. Some indicators concern for instance how the production is affected by the failure events. We will present the resilience indicator that aims at quantifying the impact of failures on the production. Other indicators are related to the gain and the risk of loss. Both types of indicator (production and gain) can be applied to one or many simulations. In the latter case, new indicators might be derived to estimate the uncertainty of the performance indicator, from its estimated variance for instance.

\subsubsection{Production resilience}

Resilience is the ability of a complex system to respond and recover from damages. The definition comes from ecology but can be found in various fields. For oil facility study, we will refer to the concept as defined in the study of urban resilience [13]. For a given simulation, resilience is used to quantify the effect of sub-optimal (i.e., disturbed) states on the level of performance. Resilience $R$ is expressed in \% and, for a discrete-time system, is computed as

$$
R=100\left(1-\frac{\left(V_{o p t}-V\right)}{V_{o p t}}\right)
$$

where $V$ is the production volume for the considered time period and $V_{o p t}$ is a reference production volume that corresponds to optimal production state. Resilience will vary from $0 \%$ (no production) to $100 \%$ (optimal production). Repeated experiments give statistical values of duration and occurrences of disturbance situations.

\subsubsection{Gain and risk of loss}

The gain represents the difference between the value of recovered oil and the operation and capital costs:

$$
\begin{array}{r}
\text { gain }=\text { recovered_oil } \times \text { barrel_price } \times \Phi_{0}-\text { OPEX } \times \Phi_{1} \\
-\quad \text { CAPEX } \times \Phi_{2}-\text { risk_losses }, \quad(27)
\end{array}
$$

where OPEX stands for operating expenditure (i.e., ongoing cost to run the field), CAPEX stands for capital expenditure (i.e., cost to acquire or upgrade the equipments) and $\Phi_{0}, \Phi_{1}, \Phi_{2}$ are factors due to income/cost sharing with consortium partners $\left(\Phi_{0}, \Phi_{1}\right.$, $\left.\Phi_{2}\right)$ or extra bank/insurance costs $\left(\Phi_{1}, \Phi_{2}\right)$.

The cumulative distribution function (cdf) of gain is denoted $F_{\text {gain }}(x)$ and defined as

$$
F_{\text {gain }}(x)=P\left(X_{\text {gain }} \leq x\right),
$$

where $P\left(X_{\text {gain }} \leq x\right)$ is the probability that the gain random variable $X_{\text {gain }}$ is smaller than $x$. The considered gain is the gain over a given time period (e.g. a week or a year if several years are simulated). Risk indicators can be computed directly from the cdf. For instance, the probability of loss can be simply estimated 


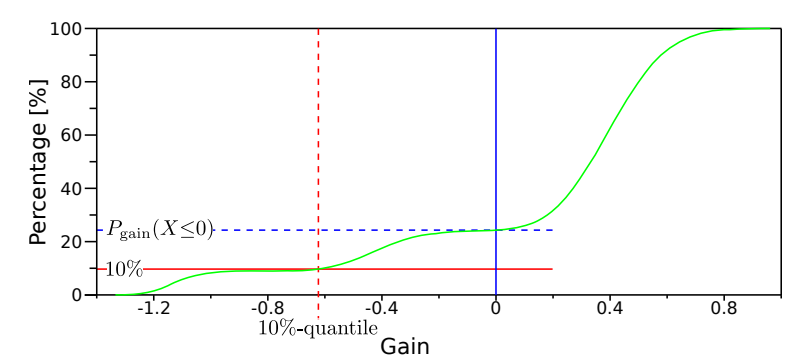

Figure 7: Estimation of the cumulative distribution function of the gain (green curve), from a one-year simulation. Intersection of the curve with the vertical line $($ Gain $=0$ ) gives an estimation of the probability of loss (blue dashed line). Intersection of the curve with the horizontal line at $10 \%$ gives an estimation of the first 10\%-quantile (red dashed line). The gain is normalized so that the maximum weekly gain is 1 .

as $F_{\text {gain }}(0)$ and the $k$-th q-quantile $(k \in[1 . . q])$ is the solution of $F_{\text {gain }}(x)=\frac{k}{q}$. Figure 7 shows the example of an estimated cdf with the estimated probability of loss and the estimated first $10 \%$-quantile.

\subsubsection{Uncertainty and performance}

All the aforementioned indicators are estimations of the real quantities that could be obtained only from an infinite number of random paths. Therefore, all decisions made on behalf of the performance indicators must include some consideration about indicator uncertainties. A tradeoff between estimated performance and reliability of the estimation should be found; for instance by representing the indicator in the space (performance, uncertainty). Monte Carlo simulation already provides insights on the variability of indicators based on the gain cdf. Indeed, confidence intervals on risk indicators can be derived as shown in Figure 8. The uncertainty can be estimated in various manners, e.g. as a function of the variance of the performance estimator. The variance can be approximated with the results of several experiments. The number $N$ of experiments can also quantify the reliability of an estimation since the latter increases with $N$.

\section{Results on a simplified design}

A simplified design has been chosen to illustrate the methodology (Figure 9). Oil is extracted from three wells whose flows are combined and sent to one among two vertical pipes (risers). The specific model for the riser is not detailed here. The two-phase flow is

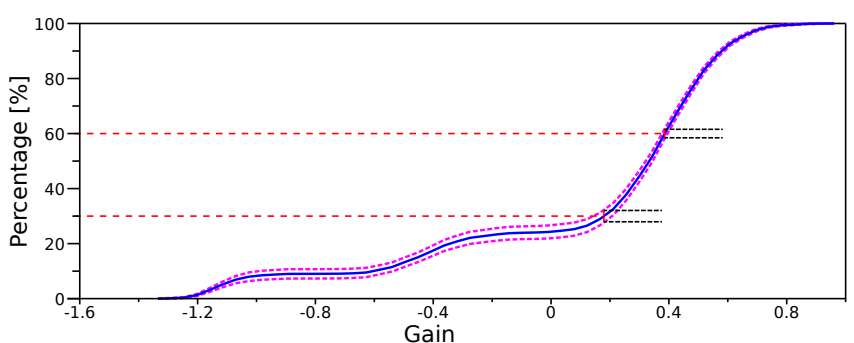

Figure 8: The cumulative distribution of gain averaged on 1000 one-year simulations (blue curve) and the confidence interval (magenta dashed curve) for a $30 \%$ and a $60 \%$ threshold. How far are the magenta curves from the blue curve tells how uncertain is the blue curve at a given time. The gain is normalized so that the maximum weekly gain -over the 1000 simulations- is 1 .

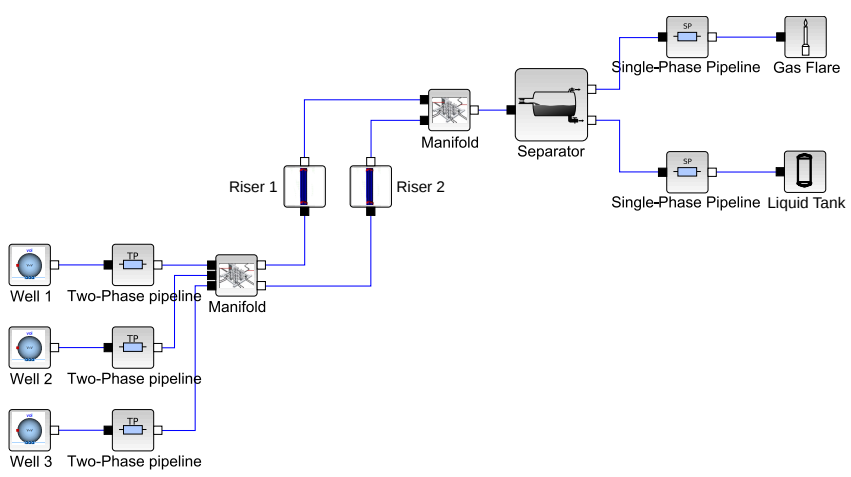

Figure 9: The studied simplified design of the offshore facility.

then received in an oil-gas separator and the two output single-phase flows are sent to a gas flare and an oil tank. The corresponding Markov chain is shown in Figure 6. States 1 and 2 are normal states with one of the two risers selected. States 3 and 4 are failure states (well 3 is blocked) with one or the other riser selected. Note that even though the characteristics (i.e., cost and production) of state 3 and 4 are identical, the states themselves are not as they do not have the same connections nor transition probabilities.

\subsection{Fluid flow simulation}

Some outputs of the fluid flow simulation are shown in Figure 10. In the presented results, initial oil level in the separator is far below the reference value, therefore all the input flow is used to fill the separator and the level in the tank does not increase until $t \approx 2000 \mathrm{~s}$. Then, because of overshoot in the controlled system, level exceeds the reference value before eventually reaching the reference. A step is added to well flow 
at $t=5000$ s that explains the little peak in oil level.
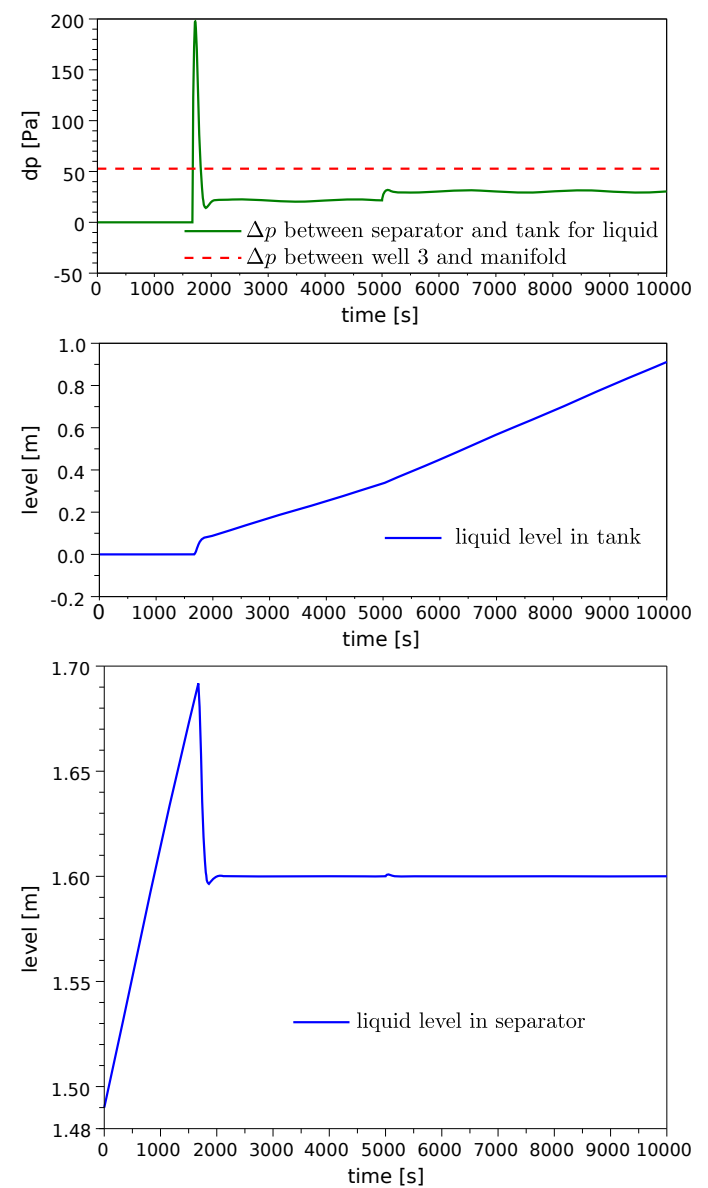

Figure 10: Some results of the fluid flow simulation with Modelica. From top to bottom: Pressure drops in a pipe with two-phase flow and at gas-oil separator outlet; oil level in final tank; oil level in separator.

\subsection{Cost and risk simulation}

Whereas all the fluid flow simulation is performed with Modelica, the cost and risk simulation uses Scilab [15]. Scilab has a built-in block diagram modeler/simulator called Scicos that can use blocks based on Modelica code. The block diagram of Figure 9 is from Scicos interface. The simulation can then be ran from a Scilab script using the scicos_simulate (...); command. Results are returned in Scilab workspace and can consequently be directly post-processed for statistical estimation. Figure 11 shows the post-processed resilience of a oneyear simulation with several computed indicators. Figure 7 is also an output of statistics processing in Scilab.

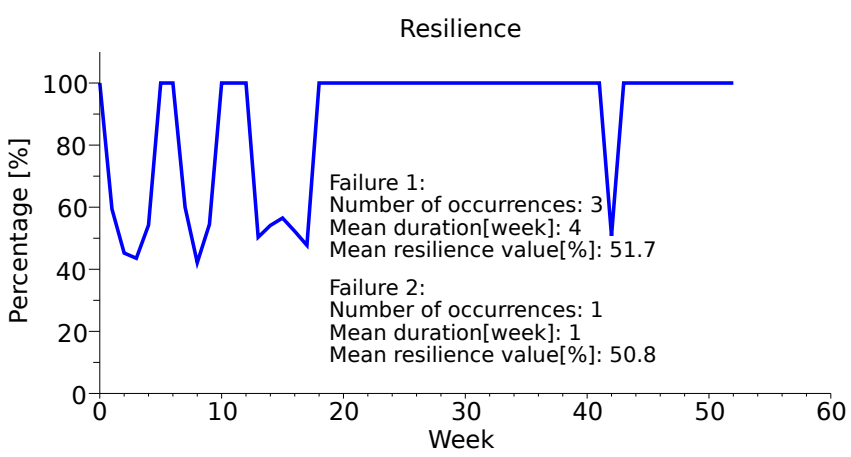

Figure 11: Resilience computed for a one-year simulation. For each type of failure, several indicators are computed like the number of occurrences or the mean duration.

\subsection{Discussion}

The presented simulation framework can be used to compare different designs of an offshore oil facility, in order to choose the most productive and/or robust, depending on the choice of a performance evaluator like those of Section 3.2.3. At the current stage of the project, the operating states and their occurring probabilities were defined arbitrarily. In further steps, they have to be set from technical data and part of the complexity of failure dependencies has to be handled automatically.

\section{Conclusion and perspectives}

We have presented our first results on the simulation of an offshore oil facility at the system level i.e., simulating all the plant components while considering also risk and failure estimations. Modelica is used to describe the physics of the flow through the various components of the offshore field. All the stochastic processes that can affect an offshore oil exploitation (failure, price variations, etc.) can be integrated in the stochastic layer of the simulation. Statistical indicators are obtained using simulation. Ongoing research is focused on up-scaling the approach and addressing industrial scale-one designs.

\section{Acknowledgments}

The authors would like to thank Arnaud Antkowiak, associate professor at Université Pierre et Marie Curie for his help on two-phase flow modeling, Jean-Louis Grange, former senior expert at EDF and Gérard Le Coq, former head of department at EDF for their support on junction models. The authors are also 
grateful to Jean-Philippe Chancelier, senior researcher at CERMICS lab of École des Ponts ParisTech, for his precious advice concerning Scilab/Scicos and its interface with Modelica. Finally, special thanks go to Lei Zhang for his several contributions to the work [17].

\section{References}

[1] Olaf Bauer. Modelling of Two-Phase Flows with Modelica. Master's thesis, Department of Automatic Control, Lund University, Sweden, November 1999.

[2] Javier Bonilla, Luis J. Yebra, Sebastián Dormido, and François E. Cellier. Object-Oriented Library of Switching Moving Boundary Models for Twophase Flow Evaporators and Condensers. In $9^{\text {th }}$ International Modelica Conference, pages 7180, September 2012.

[3] Daniel Bouskela, Audrey Jardin, Zakia Benjelloun-Touimi, Peter Aronsson, and Peter Fritzson. Modelling of uncertainties with Modelica. In $8^{\text {th }}$ International Modelica Conference, pages 673-685, March 2011.

[4] Francesco Casella, Martin Otter, Katrin Proelss, Christoph Richter, and Hubertus Tummescheit. The Modelica Fluid and Media library for modeling of incompressible and compressible thermofluid pipe networks. In $5^{\text {th }}$ International Modelica Conference, pages 631-640, September 2006.

[5] D. Chisholm. A theoretical basis for the Lockhart-Martinelli correlation for two-phase flow. International Journal of Heat and Mass Transfer, 10(12):1767-1778, 1967.

[6] Jonas Eborn and Karl Johan Åström. Modeling of a Boiler Pipe with Two-Phase Flow Instabilities. In Modelica Workshop, pages 79-88, October 2000 .

[7] EDF-EADS-PhiMeca. http://www.openturns.org/.

[8] H. Genceli, K.A. Kuenhold, O. Shoham, and J.P. Brill. Dynamic Simulation of Slug Catcher Behavior. SPE Annual Technical Conference and Exhibition, October 1988.
[9] S.E. Haaland. Simple and Explicit Formulas for the Friction Factor in Turbulent Pipe Flow. Journal of Fluids Engineering (ASME), 105(1):8990, March 1983.

[10] Jakob Munch Jensen and Hubertus Tummescheit. Moving Boundary Models for Dynamic Simulations of Two-Phase Flows. In $2^{\text {nd }}$ International Modelica Conference, pages 235-244, 2002.

[11] Lijun Ji, Bin Wu, Kui Chen, Jiawen Zhu, and Haifeng Liu. Momentum Correction Coefficient for Two Jet Flows Mixing in a Tee Junction. Chemical Engineering Research and Design, 87(8):1065-1068, 2009.

[12] R.W. Lockhart and R.C. Martinelli. Proposed Correlation of Data for Isothermal, Two-Phase Two-Component Flow in Pipes. Chemical Engineering Progress, (45):39-48, 1949.

[13] Angela Peck and Slobodan P. Simonovic. Coastal Cities at Risk (CCaR): Generic System Dynamics Simulation Models for Use with City Resilience Simulator. Technical report, Facility for Intelligent Decision Support, Department of Civil and Environmental Engineering, London, Ontario, Canada, April 2013.

[14] Gregory Provan and Alberto Venturini. Stochastic Simulation and Inference using Modelica. In $9^{\text {th }}$ International Modelica Conference, pages 829-838, September 2012.

[15] Scilab. https://www.scilab.org, (1989-2011 (INRIA) (1)1989-2007 (ENPC), Scilab Consortium (DIGITEO).

[16] Thorben Vahlenkamp and Stefan Wischhusen. FluidDissipation for Applications - A Library for Modelling of Heat Transfer and Pressure Loss in Energy Systems. In $7^{\text {th }}$ International Modelica Conference, pages 132-141, September 2009.

[17] Lei Zhang. Modélisation et Simulation d'une installation industrielle complexe. Technical report, Eurobios SCB/ENSTA ParisTech, 2013. 